\title{
Decision Support System for Online Product Recommendation Service based on Consumer Behavior
}

\author{
Sajal Acharjee \\ Britannia University \\ Comilla, Bangladesh
}

\author{
Sheikh Abujar \\ Daffodil International \\ University \\ Dhaka, Bangladesh
}

\author{
Shusa Acharjee \\ Britannia University \\ Comilla, Bangladesh
}

\author{
Shahidul Islam \\ Britannia University \\ Comilla, Bangladesh
}

\begin{abstract}
The Emerging growth of internet increases the possibility to shop over online. E-commerce facilities are increasing day by day. The rapid development of E-commerce is bound for several obligate measures. E-commerce took several liabilities for both, user and admin. Ensuring quality services demands better E-commerce product suggestions system for clients, based on their behavior. Every client should deal individual. So, product suggestions or other necessities should be done by proper analysis of every individual customer. The automatic recommendation system will reduce the extra browsing time and give customer a better experience by saving time. The system should provide personalized recommendation system. Both Supervised and unsupervised methods will be implemented to perform customer analysis for automatic recommendation system. Supervised method will be performed, only if the required customers' behaviors match with our existing large customer dataset. This will help to generate a new pattern of customer browsing history. Through this, a deep analysis could be done by our system and may use later if any customer belong to this similar pattern. An intelligent algorithm will suggest every customer, their required products list. This will help to take the decision more quickly. Ultimately, it will enhance the satisfaction rate of customers, will make this system more trust worthy and will increase the loyalty to businessman. Our research inures the customized recommendation system for every individual buyer. Several proposed methods and algorithms were stated, which will enhance the performance of existing E-commerce system and will make it more innovative and significant.
\end{abstract}

\section{Keywords}

E-Commerce, Consumer Behavior Pattern Analysis, Recommendation System, Systematic Design and Implementation, Decision Support System, Collaborative Analysis.

\section{INTRODUCTION}

The rapid development of internet availability and the accountability of faster internet services, create an opportunity for Electronic commerce (E-Commerce) system. This technology introduces faster shopping over online with the objective of saving time. The facility of choosing products online requires an important feature called Automatic product recommendation system. Over online, we could avail the befits of exploring several types of products together, at the same time. Can compare products and get instant comparison report, which helps to choose better product, as per our choice. Several times we explore the unnecessary product suggestions after frequent search. Or we may see unprovoked products suggestion as like an online advertise. After getting output of any product search, we need to see the appropriate result, where products will be competent for users. To give the customer based suggestions, algorithms have to find out the searching behavior or pattern of that customer to perform supervised method, else for unsupervised method, customers data will be measured by previous trained data set and try to found similarity based suggestions by Knn algorithm.

Different method of the recommendation system is to provide the search results by comparing the search key words, most and/or top searched products, etc. though it is not the way every user wants to have. Every customer has different choices, expecting appropriate output for individual needs to analyze the customer type and/or pattern. Through which pattern, any customer could be categorized and will provide congenial output. And it will be extensively appreciable by the customers. This facility will increase the revenue and may become able to satisfy customers. In addition, several data mining results could be explored and deployed by deep analysis of customer behavior or pattern report. Diverse product related information could be extracted and may help in further research and development.

Several recommendation system or decision support systems follow different types of methods, few are stated as: Few recommendation analysis implements following association rules, by identifying or measuring the transaction correlations. There will be a target group and another, interest group. Where both of group data will be analyzed and provide output afterwards. Basic content filtering is one of the popular methods ever used; content details will be filtered as per the information given by user. Another one is collaborative analysis, where algorithm performs by using basic topic based filtering methods and pervious user data. Through all of these methods, the recommendation or decision support system feature is still performing.

Several proposed model has drawn the attention of interactive decision support system or product recommendation system. A new Deep learning method was mentioned in the earlier of proposed models. The accuracy and performance was being measured by the generated report data with the customers expected data report. A large dataset (ICS Machine learning dataset for online retail) of user product buying records were used as a training data. Based on those data, Decisions were taken and analyzed the final report. Test results and future research scopes were explained later on this paper.

\section{LITERATURE REVIEW}

This section provides some touches about the literature overview of the product recommendation method for ecommerce and highlights the operational overview. To the enterprise and the civilization research on e-commerce recommendation has much high economic value [1]. For ordering product very fast, recommending product is very necessary. The experience product quality was changeable before consumption, so the experience products depend on 
reviews and recommendations of consumers which is theorized by Senecal et al [2]. Decision support system for product recommendation is a challenging way to attract the user to the product.

Through online product recommendation (OPR), consumer can visit or order product with less physical effort. Consumers like fewer physical effort to search in an online shopping store and searching data and comparing price are so easy [7].The practical analysis expose that higher quality of OPRs is related with higher consumer shopping efficiency which gain the loyalty of the consumers[5]. Consumer product shopping efficiency has two components, 1. Product screening efficiency 2. Product evaluation efficiency [5]. In product screening process a large amount of significant product are screened. It must not be needed to examine the level of depth. In product evaluation process, the consumers can evaluate the depth of screening product and can justify the product quality. These two types of components increase the loyalty of the product. In e-commerce, loyalty reexamines a website [6]. Product provider focuses on the consumer's loyalty which can create revenues and profitability.

Based on memory and project collaborative filtering recommendation algorithm is used [1]. Memory based collaborative filtering technology works based on the user. It works on similar users. That means If the user $\mathrm{x}$ and user $\mathrm{y}$ both are closed to behavior, we can recommend that $\mathrm{x}$ user interested in resources to y user [1].

Product type is an important part of an e-commerce online shopping site. Different products have different features. Consumer search or order product based on the features of the products. The behavior of the consumer changes with the features of the product. That means a consumer changes his mind when he see the feature of a product, though he entered for other products. And this effects on the business market strategy. For this we must take care of the ontology of the consumer's. We've to construct a Web Ontology Language (OWL) file which can store the ontology based information of the user's. This ontology information helps us to take the decision about the users. Information retrieval, artificial intelligence, knowledge network and knowledge management are gained using the ontology [3].

Overall we can reach to this decision that, to develop a meaningful online shopping website we need to attract the users or consumers by the recommended product and the features of the products. We must analyze the behavior of the consumers and who are the consumers of similar behavior. For whole operation we need large amount of data of the behavior of the users which helps us to reach to the appropriate goal.

\section{PROPOSED METHODOLOGY}

For every new user we store their information in a Web Ontology Language (OWL) file. For a semantic web which performs based on trained knowledge, ontologies play a vital role [9]. Based on this ontology information we will take decision for a user. That means the recommendation system through analyzing the user's past records and the user behavior, the product will recommend by the system. By filtering the content, comparison between user and system resources are find out to recommend the relevance of the larger resources to the user.
To overcome the customer loyalty and to take proper decision for every user we've proposed three methods. Following are the methods-
A. User Login Process for E-commerce
B. Decision Support System for product recommendation
C. Decision Support System for similar user

\subsection{User Login Process for E-commerce}

User Login System for an e-commerce is a crucial part. To keep the information of a user very safe, the login system must be very secure. At present many people are interested to online shopping system. But there are some guys who aren't interested in online shopping system because of thinking the information is vulnerable. That is why here we've discussed about the login process. The detail design of login process is stated in figure 1.

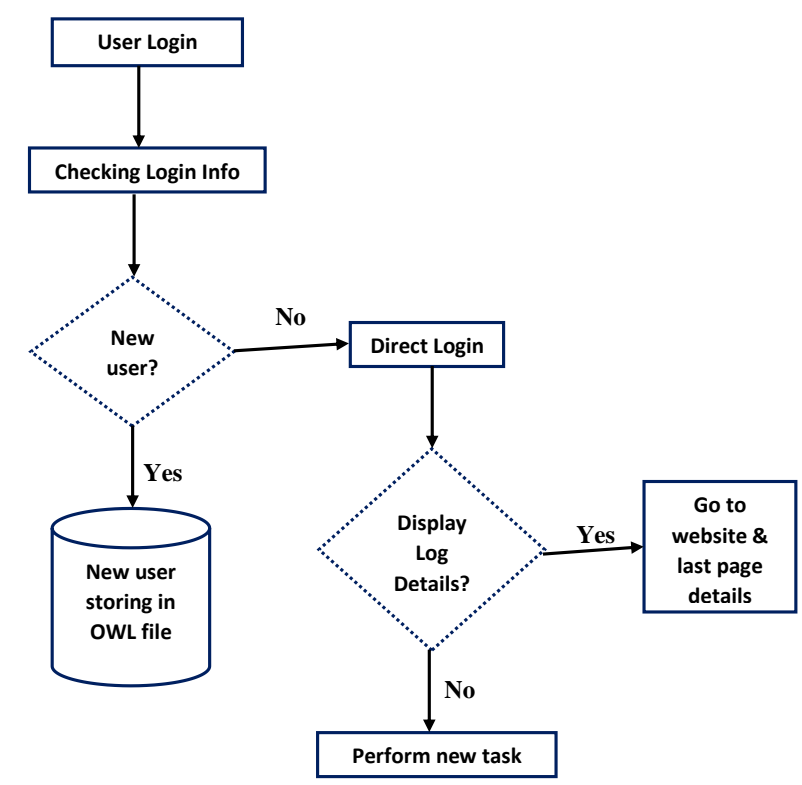

Fig. 1. Process of user login model.

In order to build up a suitable e-commerce recommendation system were drawn in figure 1 . First of all we need a perfect user login model. To verify the registered user and to cluster the user based on their behavior user must be logged into the website. At first user gives the login information and sends the request to the websites. The login methodology gives the permission to $\log$ into the webpage with checking the login info. Alongside the method also checks the user whether it is new user or not. If it is, the log of the user store into the OWL files which helps to take the decision about the user next time. If it is not, the users allowed to direct login and display the $\log$ details or perform the new task.

\subsection{Decision Support System for product recommendation:}

From the discussion till now we've known about the decision support system (DSS) all about. Here we've constructed a model about it which is drawn in figure 2, which helps us to take crucial decision for every new or registered user. The main advantage is that it tracks every user and takes immediate decision using log information. 


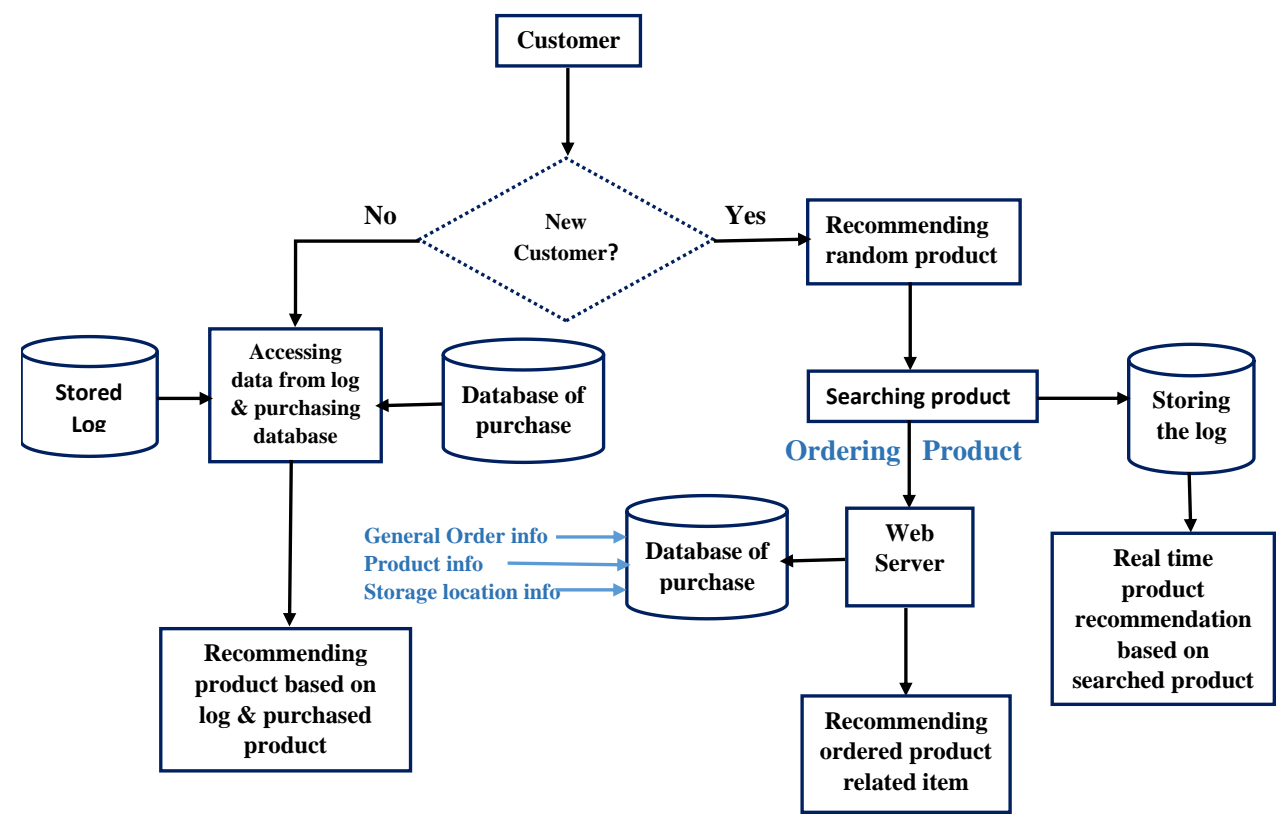

Fig. 2: DSS for Recommending Product.

In figure 2, at first it checks the customer is new or not. If it is, initially recommends random products because there is no any $\log$ information about the user. The log of the new user stores to the log database, then products will screen based on the searched products. Alongside the purchase information stores to the database of purchase product (General Order information, Product information, Storage location information) from the web server and recommends the items related to the ordered product.

If the user is registered previous user, then after login the system will access the stored $\log$ and the database of purchase. By analyzing this information that means the user's previous behavior, the system will recommend product.
This proposed model scans the user based on the log database. Using this model the provider can recommend product through the behavior of the users.

\subsection{Decision Support System for similar user:}

This paper also thinks about the similar user behavior. To analyze the user behavior more efficiently, it will be very easy if it is possible to cluster the similar user. Here we proposed a model in figure 3 , which will help to define the user whose behaviors are almost same.

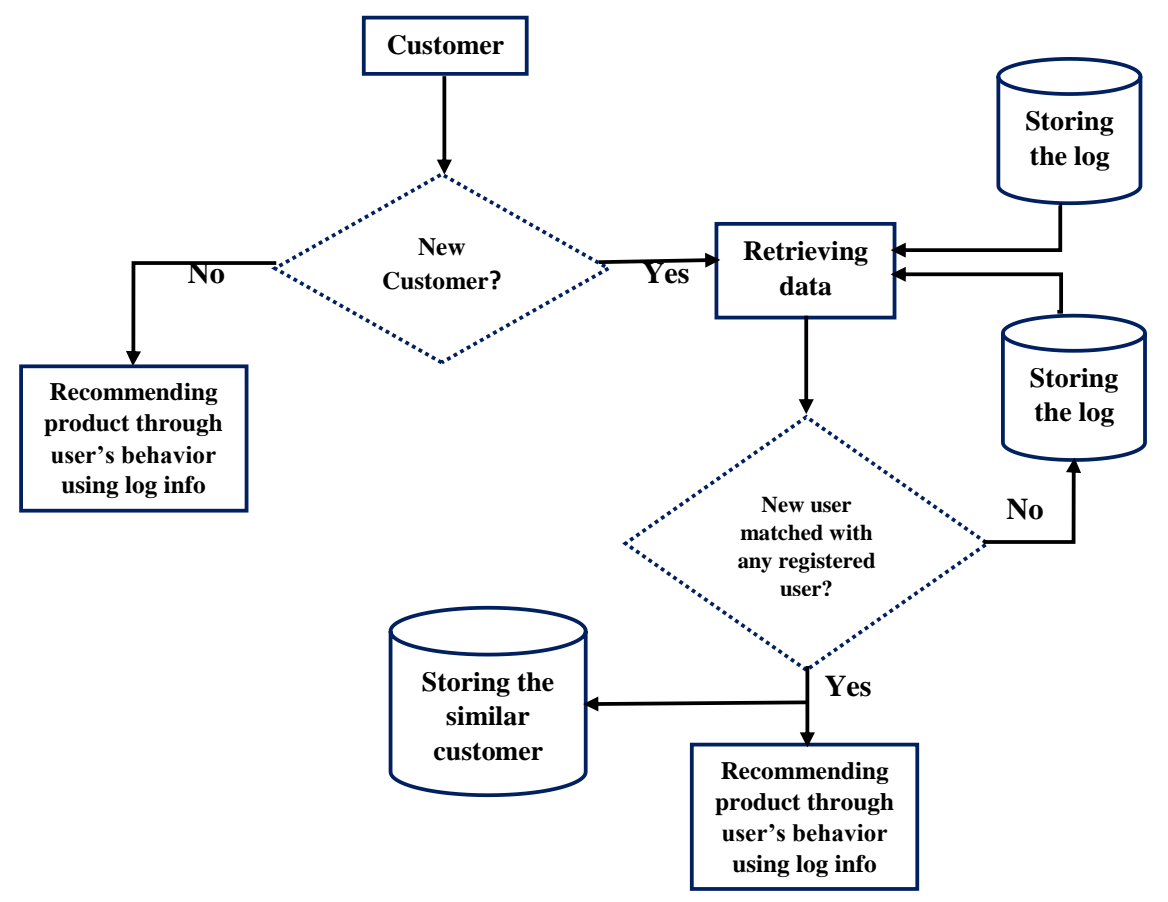

Fig. 3. Model for recommending product for similar user. 
This method works for the new user or customer. That means it retrieves from $\log$ details of new user and previous registered user to match the behavior. Then checks whether the behavior of two types of users are similar or not. If similar then the users are clustered to a class and recommends products through the behavior of the similar users. Otherwise products are recommended through the behavior of the previous registered users. The whole method uses Bayes theorem and clustering algorithm.

\section{EXPERIMENT AND RESULT}

A pilot run project was implemented based on the proposed model. A large user dataset were taken by ICS - Machine learning dataset. Where more than 56lakh user data was given. Based on comparative study of our experiments results and user given expected results (taken manually) data set, a test report was being generated. Where our system achieve $54.6 \%$ accuracy, till now. The accuracy standard was measured by comparing those two (System generated vs. user given) dataset.

\section{CONCLUSION AND FUTURE SCOPE}

Automatic recommendation or decision support system requires a huge dataset of real user, such as: data from amazon, ebay, etc service. From where we can get the original scenario of customer behavior/product search patterns for our further analysis. The efficiency may increase by categorizing users and for new users, applying the unsupervised approaches. Without appropriate customer analysis, it would be very much difficult to categorize the customers. A better text filtering algorithm can improve the research of text based product classification and user association. More case studies and validations would be very much obligate.

\section{REFERENCES}

[1] Wang, Ning, et al. "A Novel E-commerce Recommendation Algorithm based on Neural Network and Collaborative Analysis." Isi (2016): 99-104.

[2] Hassanein, Khaled, and Milena Head. "The impact of infusing social presence in the web interface: An investigation across product types." International Journal of Electronic Commerce 10.2 (2005): 31-55.

[3] Chen, Ming-Syan, Jiawei Han, and Philip S. Yu. "Data mining: an overview from a database perspective." IEEE
Transactions on Knowledge and data Engineering 8.6 (1996): 866-883.

[4] Leung, K. H., et al. "Design of a case-based multi-agent wave picking decision support system for handling ecommerce shipments." Management of Engineering and Technology (PICMET), 2016 Portland International Conference on. IEEE, 2016.

[5] Li, Xin, Jing Liu, and Fangfang Zhang. "Different effects of provider recommendations and consumer reviews on consumers' shopping efficiency for different product types." Service Systems and Service Management (ICSSSM), 2016 13th International Conference on. IEEE, 2016.

[6] Sun, Baohong. "Dynamic Structural Consumer Models and Current Marketing Issues-Comment on "Structural Modeling in Marketing: Review and Assessment"." (2005).

[7] Pavlou, Paul A., Huigang Liang, and Yajiong Xue. "Understanding and mitigating uncertainty in online environments: a principal-agent perspective." (2006)

[8] Ahmad, Manzoor, and Ms Jasira Faheem Matoo. "A Review of Ontologies Ontology Engineering and OWL for Representing Contextual Information." International Journal of Advanced Research in Computer Science and Software Engineering 3.5 (2013).

[9] Jennings, Nicholas R., Katia Sycara, and Michael Wooldridge. "A roadmap of agent research and development." Autonomous agents and multi-agent systems 1.1 (1998): 7-38.

[10] Cinicioglu, E.N., Shenoy, P.P.: A new heuristic for learning Bayesian networks fromlimited datasets: a realtime recommendation system application with RFID systems ingrocery stores. Annals of Operations Research (2012): 1-21.

[11] Zhang, Y.: CADRE: Cloud-assisted drug recommendation service for online pharmacies. Mobile Networks and Applications 20.3 (2015): 348-355.

[12] Mo, Y., Cloud-based mobile multimedia recommendation system with user behavior information. IEEE Systems Journal 8.1 (2014): 184-193. 